\title{
Indícios de eficácia dos tratamentos psicoterápicos pela internet: revisão sistemática ${ }^{1}$
}

\section{Evidences of efficacy of internet psychotherapeutic treatments: systematic review 1}

\author{
Luan Paris Feijó (orcid.org/0000-0002-7587-3987)22 \\ Ilana Luiz Fermann (orcid.org/0000-0002-0768-1211)3 \\ llana Andretta (orcid.org/0000-0002-5537-5120) ${ }^{4}$ \\ Fernanda Barcellos Serralta (orcid.org/0000-0003-4602-6495) ${ }^{5}$
}

\begin{abstract}
Resumo
A utilização das tecnologias de informação e comunicação em saúde mental é uma tendência crescente. Nos últimos anos, as pesquisas centraram-se sobre sua influência nos tratamentos psicoterápicos mediados pela internet. Este estudo realizou uma revisão sistemática de resultados dos tratamentos psicoterápicos online, a fim de verificar os indícios de sua eficácia. Buscas foram realizadas nas bases PsycINFO, Scielo e Periódicos da CAPES com os descritores "tratamento pela internet", "psicoterapia online", "telepsicologia” e "eficácia". Foram encontrados 16 estudos para análise final; os resultados indicam indícios de eficácia dessas intervenções para uma série de transtornos psicopatológicos. A terapia cognitivo comportamental foi a abordagem teórica mais utilizada nas intervenções. As intervenções online, com psicoterapeuta, foram superiores aos outros tipos de intervenções. Poucos trabalhos descreviam as características dos grupos controles e o treinamento dos psicoterapeutas. A heterogeneidade clínica e instrumentos de avaliação de resultados limitam a generalização dos achados e a comparação entre os estudos.
\end{abstract}

Palavras-chave: Revisão sistemática. Internet. Psicoterapia.

\footnotetext{
Abstract

1 O presente trabalho foi realizado com apoio da Coordenação de Aperfeiçoamento de Pessoal de Nível Superior Brasil (CAPES) - Código de Financiamento 001 e com apoio da Fundação de Amparo à Pesquisa do Estado do Rio Grande do Sul (FAPERGS).

${ }^{2}$ Factum Faculdade e Escola Técnica, Porto Alegre, Brasil. E-mail: lparisf@gmail.com.

${ }^{3}$ Universidade do Vale do Rio dos Sinos, São Leopoldo, Brasil. E-mail: ilana.fermann@gmail.com.

${ }^{4}$ Universidade do Vale do Rio dos Sinos, São Leopoldo, Brasil. E-mail: ilana.andretta@gmail.com.

${ }^{5}$ Universidade do Vale do Rio dos Sinos, São Leopoldo, Brasil. E-mail: fernandaserralta@gmail.com.
} 
The use of information and communication technologies in mental health is a growing trend. In recent years, research has focused on its influence on internet-mediated psychotherapeutic treatments. This study conducted a systematic review of the results of online psychotherapeutic treatments to verify the indications of its efficacy. Searches were performed in the databases PsycINFO, Scielo and Periódicos da CAPES with the descriptors "treatment by the internet", "online psychotherapy", "telepsychology" and "effectiveness". Sixteen studies were found for final analysis; the results indicate evidence of efficacy of these interventions for many psychopathological disorders. Cognitive-behavioral therapy was the theoretical approach most used in the interventions. Online interventions with a psychotherapist were superior to other types of interventions. Few papers described the characteristics of the control groups and the training of psychotherapists. Clinical heterogeneity and outcome assessment tools limit the generalization of findings and comparison between studies.

Keywords: Systematic review. Internet. Psychotherapy.

Processos psicoterapêuticos que utilizam tecnologias de informação e comunicação (TICs), com acesso à internet, são alvos de diversas pesquisas no contexto nacional (Crestana, 2015; Feijó, Silva, \& Benetti, 2018b; Pieta, 2014; Pieta, Siegmund, Gomes, \& Gauer, 2015; Prado \& Meyer, 2006) e internacional (Andersson et al., 2012; Barak, Hen, Boniel-Nissim, \& Shapira, 2008; Johansson et al., 2012; Rees \& Maclaine, 2015; Spek et al., 2007; Sucala et al., 2012). Atualmente, para designar as intervenções realizadas pela internet foi definido pela American Psychological Association o termo Telepsicologia (American Psychological Association, 2013; Campbell \& Norcross, 2018), em consequência da necessidade de uniformização dos distintos termos intercambiáveis utilizados (AmichaiHamburger, Klomek, Friedman, Zuckerman, \& Shani-Sherman, 2014).

No Brasil, a prática de psicoterapia por meio das TICs, utilizando a internet, foi regulamentada em 2018 pelo Conselho Federal de Psicologia (CFP), por meio da Resolução CFP n ${ }^{\circ} 11 / 2018$ (Conselho Federal de Psicologia, 2018). Anteriormente a essa resolução, estava prevista a orientação psicológica de até 20 sessões e a psicoterapia em caráter experimental (Conselho Federal de Psicologia, 2012).

Atualmente, estão disponíveis diferentes modalidades de execução dos tratamentos psicoterápicos, como complemento aos processos psicoterápicos presenciais (Feijó, Silva, \& Benetti, 2018a), por meio de intervenções baseadas na internet, geralmente estruturadas e que podem não contar com um psicoterapeuta (Barak, Klein, \& Proudfoot, 2009; Mandil, 
Bunge, Gomar, Borgialli, \& Labourt, 2009) e por fim, a psicoterapia online, com condução de um psicoterapeuta (Bunge, López, Mandil, Gomar, \& Borgialli, 2009; Pieta \& Gomes, 2014).

As psicoterapias complementadas por TICs podem ser definidas como aquelas que ocorrem durante e ao longo dos tratamentos tradicionais (Feijó et al., 2018b), sendo que, no Brasil, essa forma de intervenção é amplamente utilizada pelos psicoterapeutas, independente da abordagem psicoterápica (Feijo, Roussos, Benetti, \& Serralta, 2018). As intervenções baseadas na internet são aquelas que utilizam programas de computadores para o tratamento de distintos sintomas ou transtornos psicopatológicos, e um exemplo é o programa individual e personalizado para tratamento da depressão, chamado Deprexis (Meyer et al., 2009). Por fim, os tratamentos a distância são intervenções psicoterápicas que ocorrem online, com apoio de um psicoterapeuta e que podem ser semelhantes aos tratamentos tradicionais (Barak et al., 2009; Bunge et al., 2009).

Independente do formato de condução dessas intervenções, são necessárias as investigações sobre os seus resultados, pois embora se considere que elas apresentam resultados promissores (Machado et al., 2016; Pieta, 2014), ainda é importante avaliar em quais contextos funcionam, para quais tipos de pacientes e em quais abordagens teóricas há evidências disponíveis. Entende-se que a eficácia de um tratamento é avaliada por meio de pesquisas que envolvem ambientes controlados, como os ensaios clínicos randomizados, com amostras homogêneas e em contextos experimentais, com o objetivo de estabelecer uma relação causal entre o tratamento ofertado e a resposta obtida (Appelbaum et al., 2018; Peuker, Habigzang, Koller, \& Araujo, 2009).

Em termos de benefícios dessas intervenções, a existência de tratamentos psicoterápicos utilizando a internet poderia aumentar a procura por atendimento, devido à facilidade no acesso e por não implicar aos pacientes um alto investimento financeiro e de tempo (Backhaus et al., 2012; Proudfoot et al., 2011). No que diz respeito ao público alvo dessas intervenções, os adultos seriam aqueles que poderiam se beneficiar de tal modalidade psicoterápica e apresentarem bons resultados no tratamento, embora com restrição de quadros clínicos específicos, como nas psicoses (Carlino, 2011) e nas situações de violência e vulnerabilidade (Conselho Federal de Psicologia, 2017). 
Entretanto, a temática ainda apresenta algumas controvérsias no que diz respeito ao processo de tratamento, seja no cenário nacional ou internacional. Alguns autores consideram importantes as diferenças que existem entre o tratamento presencial das intervenções online e, dessa forma, referem que a relação terapêutica, um dos aspectos fundamentais para o sucesso da psicoterapia, poderia ficar comprometido (Machado et al., 2016; Pieta, 2014). Entre os prejuízos presumidos estão as possíveis dificuldades encontradas no estabelecimento das comunicações verbais e não verbais. Além disso, outros fatores como sigilo, identidade de pacientes e psicoterapeutas, manejos e situações de risco poderiam tornar mal sucedido o processo psicoterápico (Amichai-Hamburger et al., 2014; Kotsopoulou, Melis, Koutsompou, \& Karasarlidou, 2015).

Embora a temática seja relevante na atualidade, estudos empíricos que avaliem os atendimentos psicoterápicos pela internet ainda são escassos no Brasil (Crestana, 2015). Um dos motivos poderia residir na dificuldade em estabelecer critérios para as investigações no que diz respeito aos termos legais, éticos e aos resultados obtidos nos distintos tratamentos (Amichai-Hamburger et al., 2014; Kotsopoulou et al., 2015). Outros motivos seriam a falta de treinamento especializado entre os profissionais e o desconhecimento do uso dessas tecnologias na prática clínica (Feijó et al., 2018a). Nesse sentido, a revisão dos indícios da eficácia dos tratamentos, utilizando a internet, pode auxiliar pesquisadores e profissionais da área da saúde a conhecerem em quais situações clínicas as intervenções online são uteis para a prática clínica, pautando seu trabalho em evidências científicas.

Atualmente, existem distintas revisões sistemáticas da literatura que objetivam sistematizar a divulgação do conhecimento científico acerca do uso da internet em psicoterapia (Backhaus et al., 2012; Barak et al., 2008; Hallberg et al., 2015; Rees \& Maclaine, 2015; Spek et al., 2007; Sucala et al., 2012). De modo geral, essas revisões indicam que os estudos na terapia cognitivo comportamental são produzidos em maior escala do que em outras abordagens (Backhaus et al., 2012), que as intervenções com apoio de um agente de saúde mental moderam os resultados e são superiores às intervenções computadorizadas sem apoio (Barak et al., 2008; Spek et al., 2007) e as evidências para o tratamento dos transtornos de ansiedade são maiores do que para outras psicopatologias (Barak et al., 2008; Rees \& Maclaine, 2015). Ademais, percebe-se que a aliança terapêutica é 
igualmente bem estabelecida nesses tratamentos online, se comparada às psicoterapias presenciais (Sucala et al., 2012). Entretanto, embora bem estabelecida, questiona-se a qualidade da mesma nesses ambientes de tratamento (Sansom-Daly, Wakefield, McGill, Wilson, \& Patterson, 2016).

Portanto, considerando a necessidade de atualização clínica sobre a eficácia de tratamentos mediados pela internet e tendo como hipótese que os tratamentos presenciais podem não conseguir suprir a necessidade de atender toda a população no cenário nacional, torna-se importante investigar novas evidências clínicas para as intervenções online, como uma forma de atualizar as pesquisas clínicas na utilização da internet como meio para intervenções. Assim, o presente estudo tem como objetivo investigar os indícios de eficácia dos tratamentos psicoterápicos mediados pela internet.

\section{Método}

O artigo trata-se de uma revisão sistemática da literatura. A construção da revisão sistemática foi realizada após leitura prévia do artigo e do checklist sobre as diretrizes dos principais itens para relatar revisões sistemáticas e meta-análises [PRISMA], sendo o PRISMA utilizado no processo de construção desta revisão (Moher, Liberati, Tetzlaff, Altman, \& PRISMA Group, 2009).

Foram utilizadas duas bases de dados, PsycINFO, Scielo e o portal Periódicos da CAPES, sendo que essas bases foram escolhidas por serem as mais representativas na busca de artigos sobre a temática da revisão sistemática na área de psicologia. A pesquisa do material foi realizada com base nos últimos cinco anos, com os descritores em português e inglês: "tratamento pela internet"; "Psicoterapia Online"; "Telepsicologia" e "eficácia", utilizando o operador booleano "AND" entre os termos.

No Portal de Periódicos da CAPES, foram utilizados os filtros: (a) artigos, (b) nos últimos cinco anos, em razão da pesquisa por evidências recentes e atualizadas e (c) que contivessem os termos Psychotherapy; Clinical Trials; Randomised Controlled Trial, uma vez que o estudo da eficácia envolve delineamentos experimentais de pesquisa e ensaios clínicos (Peuker et al., 2009). No PsycINFO, as buscas foram refinadas a partir dos critérios (a) 
possuir no título a palavra internet treatment (b) em journals e (c) nos últimos cinco anos. Por fim, na base de dados Scielo, a busca conteve como filtro: (a) apenas publicações nos últimos cinco anos. A figura 1 representa os passos adotados para o processo de seleção dos artigos.

\section{Figura 1}

\section{Processo de coleta e extração dos dados}
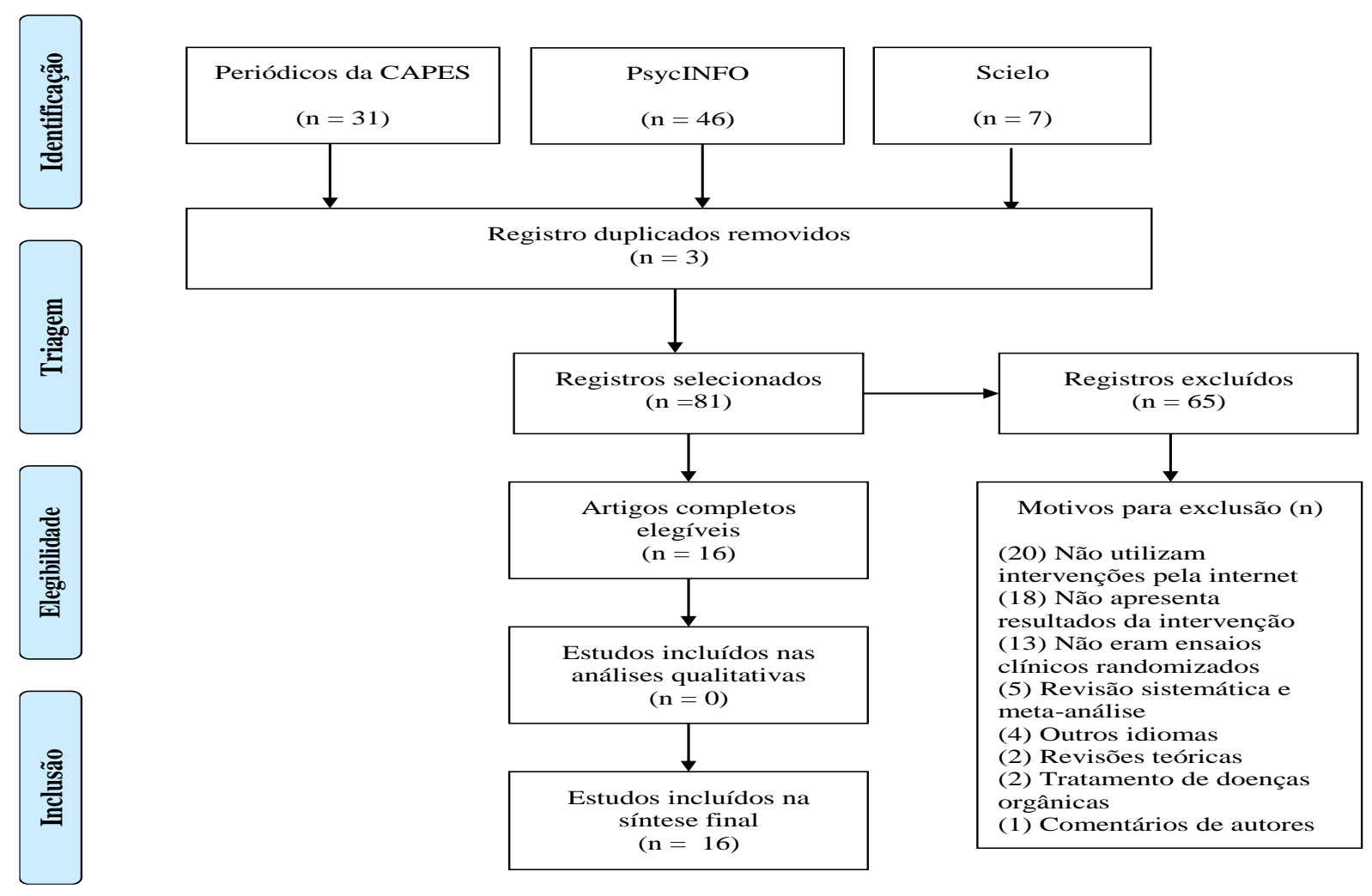

Inicialmente, foram encontrados 81 artigos. A segunda etapa foi analisar os resumos dos estudos de acordo com os seguintes critérios de inclusão: (a) referência sobre uso de tratamento psicológico mediado pela internet; (b) ensaios clínicos; (c) resultados sobre eficácia do tratamento ofertado; (d) nos idiomas português, inglês e espanhol. A aplicação desses critérios resultou na rejeição de 65 estudos. Dentre os artigos excluídos, estão: estudos que não utilizam intervenção pela internet; apresentação do protocolo de tratamento, mas sem o resultado da pesquisa; estudos que não envolviam ensaios clínicos 
randomizados, uma vez que estes são primordiais para a avaliação da eficácia de um tratamento (Peuker et al., 2009); revisões sistemáticas e meta-análise; revisões teóricas; idiomas diferentes do selecionado pelos autores; comentário de autores sobre os artigos selecionados e aqueles que fugiram do escopo do estudo, por exemplo, tratamento de doenças orgânicas pela internet e que não envolviam a temática pesquisada - intervenções psicológicas online. Assim, restaram 16 publicações para extração e análise dos materiais.

\section{Resultados e discussão}

O processo de análise dos 16 artigos foi realizado por meio de uma planilha eletrônica, por dois juízes independentes, contendo as seguintes informações: (a) autores dos estudos; (b) ano de publicação; (c) abordagem psicoterápica; (d) diagnóstico investigado; (e) características dos terapeutas; (f) características do grupo de comparação; (g) instrumentos utilizados para avaliar os resultados; (h) resultados obtidos. Nesse sentido, a Tabela 1 apresenta os dados do corpus de pesquisa contendo autores, ano e característica clínica para qual foi evidenciando a eficácia da intervenção.

\section{Tabela 1}

Descrição da síntese dos estudos extraídos

\begin{tabular}{|c|c|c|c|}
\hline $\begin{array}{c}\text { Autores e } \\
\text { ano }\end{array}$ & Abordagem & Modelo de tratamento & $\begin{array}{c}\text { Características dos } \\
\text { grupos }\end{array}$ \\
\hline $\begin{array}{l}\text { (David, } \\
\text { Schlenker, } \\
\text { Prudlo, \& } \\
\text { Larbig, } \\
\text { 2012) }\end{array}$ & $\begin{array}{l}\text { Terapia } \\
\text { Cognitiva } \\
\text { Comportamental }\end{array}$ & $\begin{array}{l}\text { Intervenção Baseada na Internet. } \\
\text { Tratamento manualizado no formato } \\
\text { psicoeducativo com elementos da TCC } \\
\text { extraídos de protocolos de tratamentos } \\
\text { existentes, em quatro módulos, com } \\
\text { duração de quatro semanas pela internet }\end{array}$ & $\begin{array}{l}\text { Estresse agudo em } \\
\text { pacientes com } \\
\text { câncer } \\
\text { hematológico }\end{array}$ \\
\hline $\begin{array}{l}\text { (Mahoney, } \\
\text { Mackenzie, } \\
\text { Williams, } \\
\text { Smith, \& } \\
\text { Andrews, } \\
\text { 2014) }\end{array}$ & $\begin{array}{l}\text { Terapia } \\
\text { Cognitiva } \\
\text { Comportamental }\end{array}$ & $\begin{array}{l}\text { Intervenção Baseada na Internet. } \\
\text { Tratamento manualizado, incluindo } \\
\text { técnicas da TCC extraídas de protocolos de } \\
\text { tratamentos existentes, em seis sessões, } \\
\text { com duração de dez semanas }\end{array}$ & $\begin{array}{l}\text { Transtorno } \\
\text { obsessivo } \\
\text { compulsivo }\end{array}$ \\
\hline $\begin{array}{l}\text { (Zernicke et } \\
\text { al., 2014) }\end{array}$ & $\begin{array}{l}\text { Terapia } \\
\text { Cognitiva }\end{array}$ & $\begin{array}{l}\text { Intervenção Baseada na Internet. } \\
\text { Tratamento manualizado, com instruções }\end{array}$ & $\begin{array}{l}\text { Sintomas de } \\
\text { estresse em }\end{array}$ \\
\hline
\end{tabular}


Comportamental didáticas, gravações guiadas e vídeos, em

e Mindfulness 16 sessões, com duração de oito semanas

(Boettcher et Terapia

al., 2014) Cognitiva

Comportamental de arquivos com exercícios, em oito

e Mindfulness

(Richards et Terapia

al., 2015) Cognitiva

Comportamental conteúdo interativo com componentes da TCC, em sete módulos.

(Rozental, Terapia Intervenção Baseada na Internet.

Forsell, Cognitiva Tratamento manualizado, incluindo a

Svensson, Comportamental utilização de técnicas da TCC, em dez

Andersson, sessões, com duração de dez semanas

\& Carlbring,

2015)

(Cernvall, Terapia Intervenção Baseada na Internet.

Carlbring, Cognitiva Tratamento manualizado, no formato de

Ljungman, Comportamental,um programa de autoajuda orientado, em

Ljungman, \& focada em

nove módulos, com duração de dez

Von Essen, psicoeducação semanas

2015)

(Fogliati et Terapia Intervenção Baseada na Internet.

al., 2016) Cognitiva Tratamento manualizado, no formato de

Comportamental tarefas online, em nove módulos, com

duração de oito semanas

(Schulz et Terapia Intervenção Baseada na Internet.

al., 2016) Cognitiva Tratamento manualizado, no formato de

Comportamental um programa baseado na TCC, em oito

sessões, com duração de 12 semanas

(Chavooshi, Psicoterapia

MohammadkDinâmica de

hani, \& Curto Prazo

Dolatshahi, Intensiva

2016)

(Wagner et Terapia

al., 2016) Cognitiva

Comportamental

Intervenção Baseada na Internet
complementada com suporte de
psicoterapeuta. Tratamento man

Intervenção Baseada na Internet,
complementada com suporte de
psicoterapeuta. Tratamento man

Psicoterapia Online. Tratamento

manualizado, no formato de psicoterapia

online, em 16 sessões, com duração de 16

semanas

incluindo terapia guiada, intensiva de

contato com terapeuta cognitivo

comportamental, em 11 módulos, com

duração de 16 semanas

(Luquiens et Terapia

al., 2016) Cognitiva
Intervenção Baseada na Internet.

Tratamento manualizado, no formato TCC, sobreviventes do

câncer

Transtorno de

ansiedade

Sintomas de

depressão

Procrastinação e

sintomas de

ansiedade

Transtorno de

stress pós-

traumático (TEPT)

Transtorno do

pânico

Transtorno de

ansiedade social

Dor e sintomas

medicamente

inexplicados

Transtorno de compulsão

alimentar

Jogo patológico 
Comportamental em seis módulos, com duração de 12 semanas

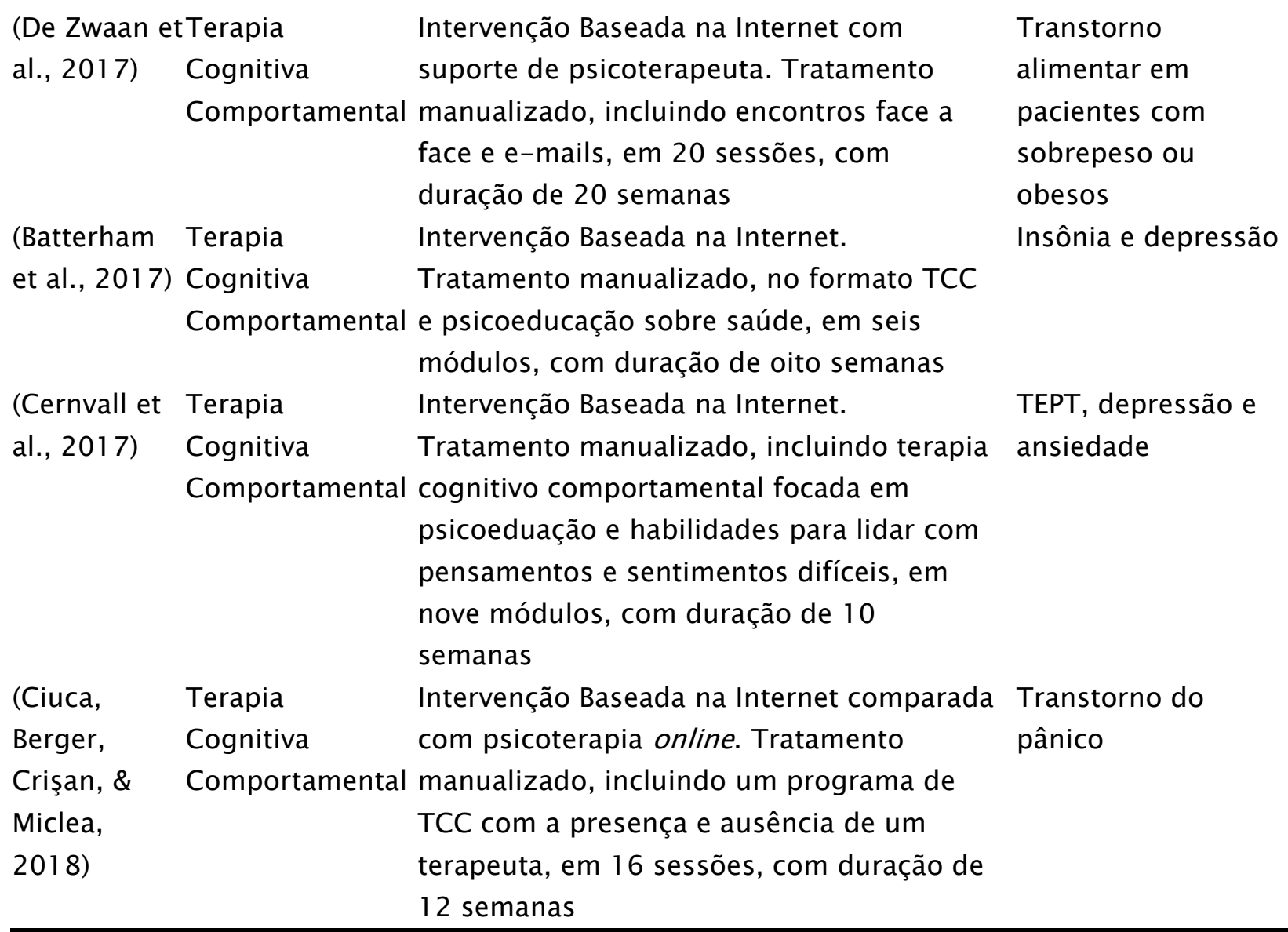

No que diz respeito ao ano dos estudos, evidenciou-se variabilidade no período de publicação, sendo 2016 o ano que mais registrou artigos disponibilizados $(n=5)$ e os anos de 2012 e 2018 com menos estudos $(n=1)$. Considerando a análise realizada a partir dos critérios estabelecidos pelos autores, os resultados apontam para a existência de vários estudos que indicam a eficácia dos tratamentos online.

No entanto, estudos que avaliavam a eficácia dos tratamentos de psicoterapia online, por videoconferência, foram escassos, nesse sentido, sinaliza-se a necessidade de estudos que envolvam essa modalidade de atendimento (Siqueira \& Russo, 2018), uma vez que este formato seria o que mais se aproximaria de uma sessão tradicional de tratamento. Ainda em relação aos estudos encontrados, verificou-se que nenhum foi realizado no Brasil, o que enfatiza a necessidade de investigação do processo e resultado psicoterápico online no âmbito nacional, considerando que o CFP autoriza e regulamenta a utilização dessa modalidade de tratamento (Conselho Federal de Psicologia, 2018). 
Em relação aos modelos de tratamentos e abordagens teóricas das intervenções, 13 estudos pautavam-se, exclusivamente, na terapia cognitivo comportamental [TCC] (Batterham et al., 2017; Cernvall et al., 2015, 2017; Ciuca et al., 2018; David et al., 2012; De Zwaan et al., 2017; Fogliati et al., 2016; Luquiens et al., 2016; Mahoney et al., 2014; Richards et al., 2015; Rozental et al., 2015; Schulz et al., 2016; Wagner et al., 2016), dois em mindfulness (Boettcher et al., 2014; Zernicke et al., 2014) e um estudo com intervenções de curta duração orientadas dinamicamente (Chavooshi et al., 2016). O panorama geral dos estudos denota quase exclusividade de abordagens vinculadas à modificação de pensamentos e comportamentos distorcidos e disfuncionais utilizando técnicas de psicoeducação e tarefas práticas, típicos de tratamentos cognitivos comportamentais, corroborando com os achados na literatura sobre a superioridade em termo de produção das intervenções em TCC nas pesquisas (Hallberg et al., 2015). Isso indica a necessidade de estudos que busquem investigar as psicoterapias a distância, principalmente na abordagem psicodinâmica (Rees \& Maclaine, 2015; Siqueira \& Russo, 2018), uma vez que essa linha teórica orienta a maioria dos psicólogos no Brasil em seu trabalho diário em psicologia clínica (Bastos, Gondim, \& Peixoto, 2010). Nesse sentido, a falta de estudos experimentais em psicoterapia psicodinâmica pode dificultar a prática pautada em evidencias por psicólogos dessa abordagem.

Quanto às características pessoais dos terapeutas, seis não descrevem essa informação no corpo do texto (Batterham et al., 2017; Boettcher et al., 2014; Chavooshi et al., 2016; David et al., 2012; De Zwaan et al., 2017; Luquiens et al., 2016), sete apenas comunicam que os mesmos estavam disponíveis para contato pelos participantes (Mahoney et al., 2014) ou possuíam treinamento e/ou realizavam supervisão (Cernvall et al., 2017; Ciuca et al., 2018; Fogliati et al., 2016; Richards et al., 2015; Rozental et al., 2015; Wagner et al., 2016). Apenas três estudos detalhavam de forma aprofundada a formação profissional ou experiência dos clínicos que conduziram as intervenções (Cernvall et al., 2015; Schulz et al., 2016; Zernicke et al., 2014). Um dos estudos caracterizou o profissional responsável pelas intervenções como especialista em oncologia (Cenvall et al., 2015), o outro incluiu uma psicóloga com mestrado em psicologia clínica e cursando o primeiro ano da especialização 
em terapia cognitivo comportamental, além de três alunas do mestrado em ciências que estavam cursando o último ano da pós-graduação em psicologia clínica e psicoterapia (Schulz et al., 2016), e, por fim, o outro estudo foi conduzido por um clínico licenciado e especializado em medicina comportamental com quinze anos de experiência no ensino online (Zernicke et al., 2014).

As informações quanto aos profissionais à frente desses tratamentos são importantes de serem destacadas nos estudos, pois podem auxiliar na problematização e na limitação dos resultados encontrados em cada um deles, uma vez que entende-se que a experiência clínica e o treinamento são fundamentais na prática do profissional, principalmente nas modalidades a distância (Carlino, 2011). Além disso, a experiência e o treinamento servem como pilares para o trabalho clínico e ignorar o efeito do terapeuta pode aumentar artificialmente o efeito da terapia, como também pode mascarar vieses do pesquisador (Crits-Christoph et al., 1991).

No que tange ao grupo de comparação dos estudos, foi possível identificar que dez deles apresentam grupo controle. No entanto, não constam as informações sobre as suas características, especificidades ou perfis (Batterham et al., 2017; Cernvall et al., 2015, 2017; Chavooshi et al., 2016; Ciuca et al., 2018; David et al., 2012; Luquiens et al., 2016; Rozental et al., 2015; Schulz et al., 2016; Wagner et al., 2016). Os outros estudos destacam as características do perfil de comparação, por exemplo, (a) que o grupo de tratamento usual (TAU) foi inscrito no programa online depois que o grupo experimental concluiu o procedimento (Mahoney et al., 2014); (b) grupo controle recebia informativos online por oito semanas (Boettcher et al., 2014); (c) lista de espera de 92 pacientes, contexto em que estes não receberam tratamento nas primeiras oito semanas e completaram as medidas de desfecho primário e secundário na oitava semana, antes de terem acesso ao programa com o apoio de um voluntário treinado (Richards et al., 2015). Por fim, dois estudos indicaram ter incluído outros tipos de intervenções para comparação, ou seja, o grupo controle recebeu outro tipo de tratamento (De Zwaan et al., 2017; Fogliati et al., 2016; Zernicke et al., 2014). As intervenções utilizadas foram descritas como GSH-I intervention e CBT intervention (De Zwaan et al., 2017), TD-TCC e TD-TD (Fogliati et al., 2016). 
Em relação ao grupo de comparação, cabe destacar que se questiona usar a lista de espera como grupo controle, uma vez que o controle de variáveis e dos eventos da vida desses sujeitos pode influenciar nos resultados do estudo e nas comparações. Ademais, grupos controles de baixa qualidade ou de tratamentos não ativos podem inflar tamanho de efeito do tratamento. No que diz respeito ao controle das variáveis e dos participantes, constatou-se que estes oscilaram consideravelmente, desde os puramente experimentais aos estudos pré experimentais, com pouco controle sobre a amostra em estudo. Embora estes últimos possam ser mais frágeis metodologicamente (Creswell, 2010), ainda assim conseguem estabelecer uma tendência dos seus resultados, como também promovem algum nível de significância clínica. Nesse sentido, discute-se que o padrão ouro para comparação da eficácia ou resultado de um tratamento seria o controle in vivo (Sansom-Daly et al., 2016).

No que diz respeito às características clínicas investigadas, os resultados apontaram para a utilização em diversos quadros psicopatológicos, sendo incompatível realizar uma análise vertical dos estudos. Contudo, os resultados sugerem benefícios para diferentes transtornos psicopatológicos que envolvam sintomas como estresse, depressão, ansiedade e insônia. Esse resultado está em consonância com os achados da literatura, indicando que os maiores tamanhos de efeito entre as psicoterapias online são para os tratamentos da ansiedade, seguidos do para a depressão (Backhaus et al., 2012; Barak et al., 2008; Rees \& Maclaine, 2015; Spek et al., 2007). Somente para jogo patológico não foram encontrados evidencias de validade superiores das intervenções online, se comparado ao tratamento usual, conforme o estudo de Luquiens et al (2016).

Para avaliação da severidade sintomática, assim como resultado da eficácia da intervenção, foram utilizados nos estudos prioritariamente escalas de autorrelato. Além dos mais, foi constatado o uso predominante de escalas de sintomas, contudo, alguns estudos incluem outras medidas - como de saúde geral ou qualidade de vida, o que é desejável para controlar os efeitos de uma intervenção, uma vez que se sugere não restringir avaliação de resultados a somente sintomas. A Tabela 2 apresenta os instrumentos de cada pesquisa e os resultados dos estudos. 


\section{Tabela 2}

Instrumentos como medida de resultado e resultado da intervenção

\begin{tabular}{|c|c|}
\hline $\begin{array}{c}\text { Autores e } \\
\text { ano }\end{array}$ & Instrumentos \\
\hline $\begin{array}{l}\text { (David et } \\
\text { al., 2012) }\end{array}$ & Brief Symptom Inventory \\
\hline $\begin{array}{l}\text { (Mahoney } \\
\text { et al., } \\
2014 \text { ) }\end{array}$ & $\begin{array}{l}\text { Dimensional Obsessive- } \\
\text { Compulsive Scale, } \\
\text { Obsessional Beliefs } \\
\text { Questionnaire-20 items, } \\
\text { Patient Health Questionnaire- } \\
9 \text { e Kessler 10-item } \\
\text { Psychological Distress scale }\end{array}$ \\
\hline
\end{tabular}

(Zernicke Profile of Mood States e et al., Calgary Symptoms of Stress

2014) Inventory

(Boettche Inventário de Depressão (BDI) $r$ et al., e Inventário de Ansiedade 2014) (BAl) pertencentes as Escalas Beck

(Richards et al., Beck - II, Questionário
Resultados

Follow-

Up

O grupo de intervenção exibiu três

aumento significativo na intenção de

se tratar ( $d=.42$; IC 95\%, 0,04 a 0,80), confirmado pela análise posterior $(\mathrm{d}$ $=.33 ; \mathrm{Cl} 95 \%, .04$ a .62).

O programa foi eficaz na redução de crenças mal adaptativas, bem como nos sintomas de TOC, angústia e depressão, com tamanho de efeito entre os grupos (>.78). A adesão ao tratamento foi alta (75\%) e os ganhos foram mantidos aos três meses de follow-up. 54\% dos que terminaram o tratamento não preenchiam mais os critérios diagnósticos para o TOC no acompanhamento.

Houve melhoria significativa, com

Não tamanho de efeito Cohen moderado no houve grupo MBCR online para os escores totais de perturbação do humor $(\mathrm{d}=.44, \mathrm{p}=.049)$, sintomas de estresse ( $d=.49, p=.021$ ) espiritualidade $(\mathrm{d}=.37, \mathrm{p}=.40)$ e atuação com consciência ( $\mathrm{d}=.50, \mathrm{p}=.026)$.

Os participantes do experimento seis mostraram diminuição maior nos meses sintomas de ansiedade, depressão e insônia na pré e pós-avaliação do que os participantes no grupo controle (d $=0,36-0,99$ ). Os tamanhos de efeito eram grandes no grupo experimental $(\mathrm{d}=.82-1,58)$ e pequeno a moderado no controle $(\mathrm{d}=.45-.76)$. Ainda, os participantes do grupo experimental alcançaram uma moderada melhoria na sua qualidade de vida.

Para o grupo de tratamento, os três e tamanhos de efeito pós-tratamento três

meses

meses seis 
2015) Sociodemográfico, General Anxiety Disorder 7 - Item Scale e Work and Social Adjustment (WSAS).

(Rozenta et al., 2015)

\section{(Cernvall} et al., 2015)

Self-report version, Inventory.

PTSD Checklist Civilian

(Fogliati et al., 2016)
(Schulz et Social Phobia Scale, Social al., 2016) Interaction Anxiety Scale, Brief Symptom Inventory e Inventário de Depressão de Beck - II relatados foram grandes para a medida meses de desfecho $(\mathrm{d}=.91)$. Os efeitos entre os grupos foram moderados a grandes e significativos para os desfechos primários $(\mathrm{d}=.50)$, favorecendo o grupo de tratamento. Os ganhos foram mantidos aos seis meses de acompanhamento.

Mudanças clinicamente significativas foram alcançadas entre $31,3 \%$ a $40 \%$ Não constav para a autoajuda dirigida, comparada a a com $24 \%$ a $36 \%$ para a autoajuda não informa guiada, $F s(98,65,17-72,55)<1,70, p$ ção $>.19$. Generalized Anxiety Disorder Assessment e Quality of Life

Análises de intenção de tratamento seis revelaram efeitos significativos em meses favor da intervenção sobre o desfecho primário de TEPT, com grandes tamanhos de efeito entre os grupos na pós-avaliação $(\mathrm{d}=.89$; IC95\% 0,35$1,43)$ e aos 12 meses de acompanhamento ( $\mathrm{d}=.78$, IC $95 \%$ 0,25-1,32). Efeitos significativos em favor da intervenção nos desfechos depressão e ansiedade também foram observados.

A intervenção indicou a redução dos sintomas de pânico $(\mathrm{d}=.71$; redução três, 12 e 24 média $\geq 36 \%$ ) e reduções moderadas a meses grandes nos sintomas de depressão comórbida $(\mathrm{d}=.71$; redução média $\geq$ 33\%), transtorno de ansiedade generalizada $(\mathrm{d}=.91$, redução média $\geq$ $34 \%)$ e transtorno de ansiedade social ( $\mathrm{d}=.50$; redução média $\geq 15 \%$ ).

No pós-tratamento, ambas as seis condições ativas apresentaram meses resultados superiores em relação aos sintomas da TAS (grupo vs. lista de espera: $\mathrm{d}=.84$ e .74 ; individual vs. 
lista de espera: $d=.94$ e 1,22).

(Chavoos Numeric Pain Rating Scale, hi et al., Depression Anxiety Stress

2016) Scale-21, Emotion Regulation Questionnaire, Mindful Attention Awareness Scale e Quality-of-Life Inventory
$\mathrm{Na}$ análise da intenção de tratar, os sintomas de dor no grupo de intervenção foram significativamente reduzidos ( $p<.001)$, onde não foi observada redução no tratamento com o grupo usual ( $p=0,651)$. Além disso, houve diminuições significativas na depressão, ansiedade e estresse, bem como um aumento no funcionamento da regulação emocional, mindfulness e qualidade de vida observada no grupo de intervenção seis meses após o tratamento.

(Wagner et al., Eating Disorder Examination Questionnaire, Inventário de

(De Zwaan et al., 2017) 2016)

(Luquiens Problem Gambling Severity et al., Index

2016) Depressão (BDI) pertencente as Escalas Beck, Symptom Checklist - 90 - Revised, peso e Índice de Massa Corporal (IMC)

2016)
Imediatamente após o tratamento, o número de episódios de compulsão alimentar mostrou melhora significativa $(d=1,02)$ no grupo de tratamento em relação à condição da lista de espera. O grupo de tratamento também reduziu significativamente os sintomas de todos os resultados de psicopatologia alimentar em relação à condição da lista de espera $(.82 \leq \mathrm{d} \leq$ 1,11 ). No grupo de tratamento, ainda foi observada melhoria significativa para todas as medidas 1 ano após a intervenção em relação aos níveis de pré-tratamento.

Todos os grupos atingiram altas taxas de atrito (83\%), mas o grupo com orientação teve uma taxa de abandono significativamente maior do que outros três grupos, incluindo o grupo controle. Embora todos os grupos tenham apresentado alguma melhora, com uma redução média de 1,35 no PGSI, não foi observada diferença significativa na eficácia entre os grupos.

A análise confirmatória usada na amostra por protocolo mostrou a não inferioridade da intervenção Online Rosenberg Self-Esteem Scale, (efeito ajustado, 1,47; 95\% Cl, -.01 a

três

seis meses

três, seis e 12 meses meses seis e 18 meses 
Impact of Weight on Quality of Life-Lite Scale, Clinical Impairment Assessment $\mathrm{e}$ Structured Clinical Interview for DSM-IV

\section{(Batterha} $m$ et al., 2017)

(Cernvall et al., 2017)
Patient Health Questionnaire9, Insomnia Severity Index (ISI), Generalized Anxiety Disorder 7-item scale e Psychiatric Symptom Frequency scale Rating Scale, Inventário de
$2,91 ; p=.05)$. Utilizando a amostra de intenção de tratar modificada, o grupo da internet foi inferior à TCC na redução dos dias de EFC no final do tratamento (efeito ajustado, 1,63; IC de 95\%, .17-3,05; $p=.03)$. Análises longitudinais exploratórias também mostraram a superioridade da TCC aos 6 meses (efeito ajustado, .36; IC 95\%, $.23-.55 ; \mathrm{p}<.001)$, mas não no seguimento de 1,5 anos (efeito ajustado, .91; IC 95\%, .54-1,50; $\mathrm{p}=.70$ ). Reduções nos achados psicopatológicos do transtorno alimentar foram significativamente maiores no grupo TCC do que no grupo internet aos seis meses de seguimento (efeito ajustado, -..4; IC95\%, -.68 a -.13; $p=.005)$.

Depressão, ansiedade e insônia diminuíram significativamente na semana 4 do período de intervenção e permaneceram significativamente menores em relação ao controle por $>$ 18 meses (entre os grupos Cohen $\mathrm{d}=$ $0,63,0,47,0,55$, respectivamente, aos 18 meses). Efeitos sobre ideação suicida foram apenas a curto prazo. Duas trajetórias de depressão foram identificadas usando modelos mistos de crescimento: melhora (95\%) e estabilidade/deterioração (5\%) dos sintomas. Depressão na linha de base mais severa, idade mais jovem e conforto limitado com a internet foram associados com chances reduzidas de melhora.

As análises de intenção de tratamento revelaram efeitos significativos em desfecho primário, com grandes Depressão de Beck - II e Inventário de Ansiedade (BAl) tamanhos de efeito entre os grupos na pós-avaliação. Efeitos significativos em favor da intervenção nos desfechos 
(Ciuca et Panic Disorder Severity Scale al., 2018) - Self Report, Psychiatric

Diagnostic Screening

Questionnaire, Patient Health

Questionnaire - 9, Work and

Social Adjustment Scale, Body

Vigilance Scale, Panic Attack

Cognition Questionnaire,

Body Sensations

Questionnaire e Patient

Feedback Questionnaire secundários depressão e ansiedade

também foram observados. No

entanto, não houve evidência de

eficácia da intervenção no consumo de assistência médica ou licença médica.

No pós-tratamento, ambas as

seis

condições ativas apresentaram

meses

resultados superiores em relação à TP

e sintomas associados (TCC orientado

vs. lista de espera: $d=1,04-1,36$; TCC

não guiado vs. Lista de espera: $d=$

.70-1,06). As duas condições ativas

não diferiram significativamente na

redução do sintoma autorrelatado $(\mathrm{d}=$ $.21-.54$, todos $\mathrm{p}>.05$ ), mas o

tratamento guiado foi superior ao

tratamento não guiado em termos de

status de diagnóstico $(X 2(1)=13,15$,

$\mathrm{p}<.01)$. Os ganhos de tratamento

foram mantidos em seguimentos

sucessivos e o tratamento guiado

tornou-se superior ao tratamento não

guiado aos seis meses de seguimento

$(\mathrm{d}=.72-1,05$, todos $\mathrm{p}<.05)$.

Os dados sinalizam que a medida avaliativa predominante nos estudos foi de autorrelato com pré-teste e pós-teste, apontando que, na maioria dos casos, os sintomas diminuiram, inclusive no periodo de follow-up. As medidas focalizam, prioritariamente, os sintomas supostamente alvos da intervenção. Porém, alguns estudos incluem medidas mais globais do ajustamento psicológico, como de qualidade de vida, por exemplo. Não foram utilizadas medidas de processos. Cabe destacar que somente um trabalho não incluiu follow-up (Rozental et al., 2015). Ademais, o período de seguimento nesses estudos variou entre três a dezoito meses.

No que tange à comparação entre as intervenções online e as presenciais, com exceção um estudo (De Zwaan et al., 2017), todos apresentaram que a intervenção online foi superior à lista de espera e aos tratamentos usuais, entretanto, no estudo citado, a intervenção presencial foi superior do que promovida pela internet. Os estudos indicam a redução de sintomas de ansiedade, depressão, intenção de se tratar e na compulsão 
alimentar, com resultados mantidos nos follow up. Ainda, os estudos destacam que as intervenções, quando guiadas por um profissional de saúde mental, demonstram-se mais promissoras do que aquelas que são auto guiadas ou não envolvem nenhum psicoterapeuta (Ciuca et al., 2018; Rozental et al., 2015).

Nesse sentido, os achados sobre o apoio de um psicoterapeuta apresentam consonância com os resultados obtidos na literatura, concluindo que o apoio terapeutico modera os resultados de um tratamento (Barak et al., 2008; Spek et al., 2007). Ainda, as pesquisas evidenciam também que a terapia por videoconferência é superior às intervenções computadorizadas (Barak et al., 2008). No presente estudo, os trabalhos que utilizaram as intervenções computadorizadas foram predominantes se comparados aos estudos com videoconferência, o que reforça a necessidade de propor pesquisas que abranjam essa especificidade de tratamento (Siqueira \& Russo, 2018).

Por fim, destaca-se que nenhum estudo incluiu alguma medida de processo ou da relação terapêutica, como a aliança terapêutica (AT), por exemplo. Isso porque, no caso da AT, embora as pesquisas indiquem não haver diferenças significativamente do seu estabelecimento entre tratamentos online e presenciais (Cook \& Doyle, 2002; Norwood, Moghaddam, Malins, \& Sabin-Farrell, 2018; Pieta, 2014; Prado \& Meyer, 2006), pouco se sabe sobre o seu desenvolvimento e processo, o que reforça a necessidade de avaliar a qualidade da mesma para compreender a adesão ou abandono nesses tipos de tratamentos.

\section{Considerações finais}

Esta revisão sistemática possibilitou identificar os estudos realizados com o objetivo de avaliar a eficácia de tratamentos psicoterápicos que utilizam as TICs. Foram encontradas algumas evidências de eficácia dessas intervenções para uma série de transtornos psicopatológicos, especialmente para os de ansiedade. A terapia cognitivo comportamental foi a abordagem teórica que mais obteve trabalhos publicados nos últimos cinco anos. As intervenções online, pela internet, com apoio de um agente de saúde mental foram superiores às intervenções sem apoio e aos tratamentos usuais, em quase todos os estudos. 
Todos os trabalhos mediram os resultados do tratamento por meio de escalas de autorrelato que avaliavam sintomas específicos da condição clínica para a qual a intervenção estava destinada. Raros trabalhos descreviam as características dos grupos controles e o treinamento dos psicoterapeutas. Das características dos estudos revisados, esta última talvez seja a principal limitação, pois se avalia a terapia como se ela fosse independente do terapeuta e produzisse efeito per se. Por fim, somente foi localizado um estudo utilizando videoconferência e em modelo de psicoterapia psicodinâmica, o que reforça a necessidade de investigação nessa abordagem teórica.

Destaca-se a necessidade de realização de estudos nacionais que tratem dessa temática e que possam avaliar não só a eficácia, mas também o impacto dessas intervenções nos indivíduos e nos processos de tratamento, uma vez que, no Brasil, a psicoterapia utilizando intervenções pela internet é recente, aprovada em 2018, e carece de dados empíricos. Dessa forma, necessita-se avançar as pesquisas em psicoterapia centrando-se não somente no resultado final de um tratamento, mas também no que ocorre ao longo do processo, uma vez que as mudanças psicoterapeuticas podem estar focalizadas em sessões específicas, sendo que essas situações são importantes no tratamento e na sua evolução, e podem não aparecer no resultado final (Peuker et al., 2009; Serralta, Nunes, \& Eizirik, 2011). Além disso, considera-se relevante, também, que as pesquisas envolvam outras abordagens teóricas, principalmente das orientadas dinamicamente, que os ensaios clínicos randomizados sejam realizados com grupos controles in vivo, que haja melhor descrição no que diz respeito à formação e treinamento dos psicoterapeutas e, por fim, que os estudos incluam medidas globais e não apenas de sintomas que avaliem pré-intervenção e pósintervenção.

Pode-se destacar como limitadores deste estudo: a exclusão de dissertações, teses e estudos ainda não publicados em revistas avaliadas pelos pares em psicologia; ainda, não se avaliou, as informações sobre a adesão ou abandono nesses tratamentos, o que reforça a necessidade de investigar esses dados por outros pesquisadores. Ademais, optou-se por um período específico de publicação, pois as TICs sofrem modificações e atualizações, o que pode não ter contemplado estudos realizados anteriormente. Por fim, seria importante incluir bases de dados que não sejam somente da ciência psicológica e o uso de descritores 
em espanhol. Enquanto direções futuras, sugere-se a avaliação de estudos de efetividade e de processo psicoterapêutico nas intervenções psicológicas online, especialmente no contexto brasileiro, considerando que o contexto social pode influenciar nos resultados do tratamento psicológico.

\section{Referências}

American Psychological Association. (2013). Guidelines for the practice of telepsychology. American Psychologist, 68(9), 791-800. https://doi.org/10.1037/a0035001

Amichai-Hamburger, Y., Klomek, A. B., Friedman, D., Zuckerman, O., \& Shani-Sherman, T. (2014). The future of online therapy. Computers in Human Behavior, 41, 288-294. https://doi.org/10.1016/j.chb.2014.09.016

Andersson, G., Paxling, B., Roch-Norlund, P., Stman, G., Norgren, A., Almlov, J. et al. (2012). Internet-based psychodynamic versus cognitive behavioral guided self-help for generalized anxiety disorder: A randomized controlled trial. Psychotherapy and Psychosomatics, 87(6), 344-355. https://doi.org/10.1159/000339371

Appelbaum, M., Cooper, H., Kline, R. B., Mayo-Wilson, E., Nezu, A. M., \& Rao, S. M. (2018). Reporting Standards for Qualitative Research in Psychology: The APA Publications and Communications Board Task Force Report. American Psychologist, 73(1), 3-25. https://doi.org/10.1037/amp0000151

Backhaus, A., Agha, Z., Maglione, M. L., Repp, A., Ross, B., Zuest, D. et al. (2012). Videoconferencing psychotherapy: a systematic review. Psychological Services, 9(2), 111-131. https://doi.org/10.1037/a0027924

Barak, A., Hen, L., Boniel-Nissim, M., \& Shapira, N. (2008). A Comprehensive Review and a Meta-Analysis of the Effectiveness of Internet- Based Psychotherapeutic Interventions. Journal of Technology in Human Services, 26(2/4), 109-160. https://doi.org/10.1080/15228830802094429

Barak, A., Klein, B., \& Proudfoot, J. G. (2009). Defining internet-supported therapeutic interventions. Annals of Behavioral Medicine, 38(1), 4-17. https://doi.org/10.1007/s12160-009-9130-7

Bastos, A. V. B., Gondim, S. M. G., \& Peixoto, L. S. A. (2010). Áreas de atuação, atividades e abordagens teóricas do psicólogo brasileiro. In A. V. B. Bastos \& S. M. G. Gondim (Orgs.), O Trabalho do Psicólogo no Brasil (p. 502). Porto Alegre: Artmed.

Batterham, P. J., Christensen, H., Mackinnon, A. J., Gosling, J. A., Thorndike, F. P., Ritterband, L. M. et al. (2017). Trajectories of change and long-term outcomes in a randomised controlled trial of internet-based insomnia treatment to prevent depression. British Journal of Psychiatry Open, 3(5), 228-235. https://doi.org/10.1192/bjpo.bp.117.005231

Boettcher, J., Åström, V., Påhlsson, D., Schenström, O., Andersson, G., \& Carlbring, P. (2014). Internet-Based Mindfulness Treatment for Anxiety Disorders: A Randomized Controlled 
Trial. Behavior Therapy, 45(2), 241-253. https://doi.org/10.1016/j.beth.2013.11.003

Bunge, E., López, P., Mandil, J., Gomar, M., \& Borgialli, R. (2009). Actitudes de los Terapeutas Argentinos hacia la Incorporación de Nuevas Tecnologías en Psicoterapia. Revista Argentina de Clínica Psicológica, 18, 209-216. http://www.redalyc.org/articulo.oa?id $=281921775002$

Campbell, L. F., \& Norcross, J. C. (2018). Do you see what we see? Psychology's response to technology in mental health. Clinical Psychology: Science and Practice, e12237, 1-5. https://doi.org/10.1111/cpsp.12237

Carlino, R. (2011). Distance Psychoanalysis: The Theory and Practice of using Communication Technology in the Clinic. London: Karnac Books.

Cernvall, M., Carlbring, P., Ljungman, L., Ljungman, G., \& Von Essen, L. (2015). Internetbased guided self-help for parents of children on cancer treatment: $A$ randomized controlled trial. Psycho-Oncology, 1158 (March), $1152-1158$. https://doi.org/http://dx.doi.org/10.1002/pon.3874

Cernvall, M., Carlbring, P., Wikman, A., Ljungman, L., Ljungman, G., \& Von Essen, L. (2017). Twelve-month follow-up of a randomized controlled trial of internet-based guided selfhelp for parents of children on cancer treatment. Journal of Medical Internet Research, 19(7), 1-12. https://doi.org/10.2196/jmir.6852

Chavooshi, B., Mohammadkhani, P., \& Dolatshahi, B. (2016). A Randomized Double-Blind Controlled Trial Comparing Davanloo Intensive Short-Term Dynamic Psychotherapy as Internet-Delivered Vs Treatment as Usual for Medically Unexplained Pain: A 6-Month $\begin{array}{lll}\text { Pilot Study. } & \text { Psychosomatics, 292-300. }\end{array}$ https://doi.org/10.1016/j.psym.2016.01.001

Ciuca, A. M., Berger, T., Crişan, L. G., \& Miclea, M. (2018). Internet-based treatment for panic disorder: A three-arm randomized controlled trial comparing guided (via real-time video sessions) with unguided self-help treatment and a waitlist control. PAXPD study results. Journal of Anxiety Disorders, (March). https://doi.org/10.1016/j.janxdis.2018.03.009

Conselho Federal de Psicologia. (2012). Resolução CFP N $011 / 2012$. Regulamenta os serviços psicológicos realizados por meios tecnológicos de comunicação a distância, o atendimento psicoterapêutico em caráter experimental e revoga a Resolução CFP $\mathrm{N}^{\circ}$ 12/2005. Brasília, DF: CPF. http://site.cfp.org.br/wpcontent/uploads/2012/07/Resoluxo_CFP_nx_011-12.pdf

Conselho Federal de Psicologia. (2018). Resolução No 11, de 11 de maio de 2018. Brasília, DF: CPF. https://site.cfp.org.br/wp-content/uploads/2018/05/RESOLUÇÃO-No-11-DE11-DE-MAIO-DE-2018.pdf

Conselho Federal de Psicologia. (2017, Dezembro 17). Resolução do Conselho Federal de Psicologia regulamenta prática on-line. Conselho Federal de Psicologia. https://site.cfp.org.br/resolucao-do-cfp-regulamenta-pratica-on-line/

Cook, J. E., \& Doyle, C. (2002). Working Alliance in Online Therapy as Compared to Face-toFace Therapy: Preliminary Results. CyberPsychology \& Behavior, 5(2), 95-105. https://doi.org/10.1089/109493102753770480

Crestana, T. (2015). Novas abordagens terapêuticas - terapias on-line. Revista Brasileira de Psicoterapia, 17(2), 35-43. http://rbp.celg.org.br/detalhe_artigo.asp?id=176

Creswell, J. W. (2010). Projeto de pesquisa: Métodos qualitativos, quantitativos e mistos (3a 
ed). Porto Alegre: Artmed. http://loja.grupoa.com.br/livros/pesquisa-cientifica/projetode-pesquisa/9788536323008

Crits-Christoph, P., Baranackie, K., Kurcias, J. S., Beck, A. T., Carroll, K., Perry, K. et al. (1991). Meta-analysis of therapist effects in psychotherapy outcome studies. Psychotherapy Research, 1(2), 81-91. https://doi.org/10.1080/10503309112331335511

David, N., Schlenker, P., Prudlo, U., \& Larbig, W. (2012). Internet-based program for coping with cancer: A randomized controlled trial with hematologic cancer patients. PsychoOncology, (December 2010). https://doi.org/10.1002/pon.3104

De Zwaan, M., Herpertz, S., Zipfel, S., Svaldi, J., Friederich, H. C., Schmidt, F. et al. (2017). Effect of internet-based guided self-help vs individual face-to-face treatment on full or subsyndromal binge eating disorder in overweight or obese patients: The INTERBED randomized clinical trial. JAMA Psychiatry, 74(10), 987-995. https://doi.org/10.1001/jamapsychiatry.2017.2150

Feijo, L. P., Roussos, A. J., Benetti, S. P. C., \& Serralta, F. B. (2018). Uso de las tecnologías de información y comunicación en el contexto brasileño de psicoterapia. Anales 130 Congreso Latinoamericano De Investigacion En Psicoterapia SPR y $15^{\circ}$ Congreso Chileno De Psicoterapia. http://congresopsicoterapia.cl/Programafinal2018.pdf

Feijó, L. P., Silva, N. B., \& Benetti, S. P. C. (2018a). Experiência e formação profissional de psicoterapeutas psicanalíticos na utilização das tecnologias de informação e comunicação. Psicologia: Ciência e Profissão, 38(2), 249-261. https://doi.org/10.1590/1982-3703003032017

Feijó, L. P., Silva, N. B., \& Benetti, S. P. C. (2018b). Impacto das tecnologias de informação e comunicação na técnica psicoterápica psicanalítica. Temas em Psicologia, 26(3), 16331647. https://doi.org/10.9788/TP2018.3-18En

Fogliati, V. J., Dear, B. F., Staples, L. G., Terides, M. D., Sheehan, J., Johnston, L. et al. (2016). Disorder-specific versus transdiagnostic and clinician-guided versus self-guided internet-delivered treatment for panic disorder and comorbid disorders: A randomized controlled trial. Journal of Anxiety Disorders, 39, 88-102. https://doi.org/10.1016/j.janxdis.2016.03.005

Hallberg, S. C. M., Lisboa, C. S. M., Souza, D. B., Mester, A., Braga, A. Z., Strey, A. M. et al. (2015). Systematic review of research investigating psychotherapy and information and communication technologies Revisão sistemática sobre psicoterapia e tecnologias da informação e comunicação. Trends Psychiatry Psychother, 37(3), 118-125. https://doi.org/10.1590/2237-6089-2014-0055

Johansson, R., Ekbladh, S., Hebert, A., Lindström, M., Möller, S., Petitt, E. et al. (2012). Psychodynamic guided self-help for adult depression through the internet: $A$ randomised controlled trial. PLOS ONE, $7(5), \quad 1-9$. https://doi.org/10.1371/journal.pone.0038021

Kotsopoulou, A., Melis, A., Koutsompou, V. I., \& Karasarlidou, C. (2015). E-therapy: The Ethics behind the Process. Procedia Computer Science, 65, 492-499. https://doi.org/10.1016/j.procs.2015.09.120

Luquiens, A., Tanguy, M. L., Lagadec, M., Benyamina, A., Aubin, H. J., \& Reynaud, M. (2016). The efficacy of three modalities of internet-based psychotherapy for non-treatment- 
seeking online problem gamblers: A randomized controlled trial. Journal of Medical Internet Research, 18(2), 1-13. https://doi.org/10.2196/jmir.4752

Machado, D. de B., Laskoski, P. B., Severo, C. T., Bassols, A., Sfoggia, A., Kowacs, C. et al. (2016). A Psychodynamic Perspective on a Systematic Review of Online Psychotherapy for Adults. British Journal of Psychotherapy, 32(1), 79-108. https://doi.org/10.1111/bjp.12204

Mahoney, A. E. J., Mackenzie, A., Williams, A. D., Smith, J., \& Andrews, G. (2014). Internet cognitive behavioural treatment for obsessive compulsive disorder: A randomised controlled trial. Behaviour Research and Therapy, 63, 99-106. https://doi.org/10.1016/j.brat.2014.09.012

Mandil, J., Bunge, E., Gomar, M., Borgialli, R., \& Labourt, J. (2009). La implementación de recursos tecnológicos en la clínica con niños y adolescentes. Revista Argentina de Clínica Psicológica, 18(1), 59-68. http://www.redalyc.org/articulo.oa?id=281921800006\%0ACómo

Meyer, B., Berger, T., Caspar, F., Beevers, C. G., Andersson, G., \& Weiss, M. (2009). Effectiveness of a novel integrative online treatment for depression (Deprexis): randomized controlled trial. Journal of medical Internet research, 17(2), e15. https://doi.org/10.2196/jmir.1151

Moher, D., Liberati, A., Tetzlaff, J., Altman, D. G., \& PRISMA Group. (2009). Preferred Reporting Items for Systematic Reviews and Meta-Analyses: The PRISMA Statement. PLoS Medicine, 6(7), e1000097. https://doi.org/10.1371/journal.pmed.1000097

Norwood, C., Moghaddam, N. G., Malins, S., \& Sabin-Farrell, R. (2018). Working alliance and outcome effectiveness in videoconferencing psychotherapy: A systematic review and noninferiority meta-analysis. Clinical Psychology \& Psychotherapy, (June), 1-12. https://doi.org/10.1002/cpp.2315

Peuker, A. C., Habigzang, L. F., Koller, S. H., \& Araujo, L. B. (2009). Avaliação de processo e resultado em psicoterapias: Uma revisão. Psicologia em Estudo, 14(3), 439-445. https://doi.org/10.1590/S1413-73722009000300004

Pieta, M. A. M. (2014). Psicoterapia Pela Internet: a Relação Terapêutica [Tese de Doutorado, Universidade Federal do Rio Grande do Sul]. LUME Repositório Digital UFRGS. http://hdl.handle.net/10183/144025

Pieta, M. A. M., \& Gomes, W. B. (2014). Psicoterapia pela internet: Viável ou inviável? Psicologia: Ciência e Profissâo, 34(1), 18-31. https://doi.org/10.1590/S1414$\underline{98932014000100003}$

Pieta, M. A. M., Siegmund, G., Gomes, W. B., \& Gauer, G. (2015). Desenvolvimento de protocolos para acompanhamento de psicoterapia pela Internet. Contextos Clínicos, 8(2), 128-140. https://doi.org/10.4013/ctc.2015.82.02

Prado, O., \& Meyer, S. (2006). Avaliação da relação terapêutica na terapia assíncrona via internet. Psicologia em Estudo, 17(2), 247-257. https://doi.org/10.1590/S141373722006000200003

Proudfoot, J., Klein, B., Barak, A., Carlbring, P., Cuijpers, P., Lange, A. et al. (2011). Establishing guidelines for executing and reporting internet intervention research. $\begin{array}{llll}\text { Cognitive Behaviour } & \text { Therapy, } & \text { 82-97. }\end{array}$ https://doi.org/10.1080/16506073.2011.573807 
Rees, C. S., \& Maclaine, E. (2015). A Systematic Review of Videoconference-Delivered Psychological Treatment for Anxiety Disorders. Australian Psychologist, 50(4), 259-264. https://doi.org/10.1111/ap.12122

Richards, D., Timulak, L., O'Brien, E., Hayes, C., Vigano, N., Sharry, J. et al. (2015). A randomized controlled trial of an internet-delivered treatment: Its potential as a lowintensity community intervention for adults with symptoms of depression. Behaviour Research and Therapy, 75, 20-31. https://doi.org/10.1016/j.brat.2015.10.005

Rozental, A., Forsell, E., Svensson, A., Andersson, G., \& Carlbring, P. (2015). Internet-Based Cognitive- Behavior Therapy for Procrastination: A Randomized Controlled Trial. Journal of Consulting and Clinical Psychology, 83(4), 808-824. https://doi.org/10.1037/ccp0000023

Sansom-Daly, U. M., Wakefield, C. E., McGill, B. C., Wilson, H. L., \& Patterson, P. (2016). Consensus Among International Ethical Guidelines for the Provision of Videoconferencing-Based Mental Health Treatments. JMIR mental health, 3(2), el 7. https://doi.org/10.2196/mental.5481

Schulz, A., Stolz, T., Vincent, A., Krieger, T., Andersson, G., \& Berger, T. (2016). A sorrow shared is a sorrow halved? A three-arm randomized controlled trial comparing internetbased clinician-guided individual versus group treatment for social anxiety disorder. Behaviour Research and Therapy, 84, 14-26. https://doi.org/10.1016/j.brat.2016.07.001

Serralta, F. B., Nunes, M. L. T., \& Eizirik, C. L. (2011). Considerações metodológicas sobre o estudo de caso na pesquisa em psicoterapia. Estudos de Psicologia (Campinas), 28(4), 501-510. https://doi.org/10.1590/S0103-166X2011000400010

Siqueira, C. C. A., \& Russo, M. N. (2018). Psicoterapia on-line: ética, segurança e evidências científicas sobre práticas clínicas mediadas por tecnologias (1a ed.). São Paulo: Zagodoni.

Spek, V., Cuijpers, P., Nyklícek, I., Riper, H., Keyzer, J., \& Pop, V. (2007). Internet-based cognitive behaviour therapy for symptoms of depression and anxiety: a meta-analysis. Psychological Medicine, 37(03), 319. https://doi.org/10.1017/S0033291706008944

Sucala, M., Schnur, J. B., Constantino, M. J., Miller, S. J., Brackman, E. H., \& Montgomery, G. H. (2012). The therapeutic relationship in E-therapy for mental health: A systematic review. Journal of Medical Internet Research, 14(4), e110. https://doi.org/10.2196/jmir.2084

Wagner, B., Nagl, M., Dölemeyer, R., Klinitzke, G., Steinig, J., Hilbert, A. et al. (2016). Randomized Controlled Trial of an Internet-Based Cognitive-Behavioral Treatment Program for Binge-Eating Disorder. Behavior therapy, 47(4), 500-514. https://doi.org/10.1016/j.beth.2016.01.006

Zernicke, K. A., Campbell, T. S., Speca, M., Mccabe-Ruff, K., Flowers, S., \& Carlson, L. E. (2014). A randomized wait-list controlled trial of feasibility and efficacy of an online mindfulness-based cancer recovery program: The eTherapy for cancer applying mindfulness trial. Psychosomatic Medicine, 76(4), 257-267. https://doi.org/10.1097/PSY.0000000000000053 
Feijó, L. P. et al.

Página 25 de 25

Recebido em: 5/9/2018

Aprovado em: 15/11/2018 